\title{
Aspergillus-related lung disease in people with cystic fibrosis: can imaging help us to diagnose disease?
}

\author{
Qianting Lv ${ }^{1,2}$, Bernadette B.L.J. Elders ${ }^{1,2}$, Adilia Warris ${ }^{3}$, Daan Caudri ${ }^{1}$, Pierluigi Ciet ${ }^{1,2}$ and \\ Harm A.W.M. Tiddens $\mathbb{1}^{1,2}$
}

${ }^{1}$ Dept of Paediatric Pulmonology and Allergology, Erasmus Medical Centre - Sophia Children's Hospital, Rotterdam, The Netherlands. ${ }^{2}$ Dept of Radiology and Nuclear Medicine, Erasmus Medical Centre, Rotterdam, The Netherlands. ${ }^{3}$ MRC Centre for Medical Mycology, University of Exeter, Exeter, UK.

Corresponding author: Harm A.W.M. Tiddens (h.tiddens@erasmusmc.nl)

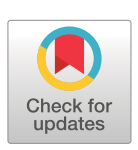

This version is distributed under the terms of the Creative Commons Attribution NonCommercial Licence 4.0. For commercial reproduction rights and permissions contact permissions@ersnet.org

Received: 23 April 2021 Accepted: 7 Aug 2021

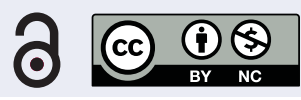

Shareable abstract (@ERSpublications)

Specific structural lung damage on chest CT and MRI associated with $A$. fumigatus infection in CF can be identified. A. fumigatus-related structural lung damage can be detected on images at an early stage to guide treatment and clinical management in CF. https://bit.ly/3CWzQWq

Cite this article as: Lv Q, Elders BBLJ, Warris A, et al. Aspergillus-related lung disease in people with cystic fibrosis: can imaging help us to diagnose disease? Eur Respir Rev 2021; 30: 210103 [DOI: 10.1183/ 16000617.0103-2021].

\section{Abstract}

In people with cystic fibrosis (PwCF), viscous sputum and dysfunction of the mucociliary escalator leads to early and chronic infections. The prevalence of Aspergillus fumigatus in sputum is high in PwCF and the contribution of A. fumigatus to the progression of structural lung disease has been reported. However, overall, relatively little is known about the contribution of A. fumigatus to CF lung disease. More knowledge is needed to aid clinical decisions on whether to start antifungal treatment. In this review, we give an overview of A. fumigatus colonisation and infection in PwCF and the different types of pulmonary disease caused by it. Furthermore, we discuss the current evidence for structural lung damage associated with A. fumigatus in PwCF on chest computed tomography and magnetic resonance imaging. We conclude that radiological outcomes to identify disease caused by $A$. fumigatus can be important for clinical studies and management.

\section{Introduction}

Cystic fibrosis (CF) is a multi-system disease caused by a mutation in the gene that encodes for the cystic fibrosis transmembrane conductance regulator (CFTR) protein [1-3]. The CFTR protein defect leads to an abnormal composition of the epithelial lining fluid, viscous sputum and dysfunction of the mucociliary escalator [3-5]. As a result, most people with CF (PwCF) suffer from early and chronic airway infections caused by a wide range of microorganisms, especially Staphylococcus aureus, Pseudomonas aeruginosa and Aspergillus fumigatus [6-10].

There is a huge amount of knowledge available about bacterial infections in PwCF, but relatively little is known about the contribution of $A$. fumigatus infection. The prevalence of $A$. fumigatus-positive sputum cultures in adult PwCF ranges from $27 \%$ to $57 \%$ [11]. The prevalence of $A$. fumigatus in paediatric PwCF was thought to be relatively low under the age of 8 years, and more common in children older than 10 years [12]. However, the incidence may be significantly underestimated in children [13]. In 2018 and 2019, studies using bronchoalveolar lavage (BAL) samples taken from children up to the age of 6 years found the median age of colonisation with Aspergillus species (mainly A. fumigatus) in PwCF to be 3.2 years [14], and $A$. fumigatus to be present in up to $28 \%$ of patients from the age of 3 years onwards [15].

Previous research has indicated that $A$. fumigatus is not an innocent bystander but can contribute substantially to structural lung damage [11, 15-23]. However, it is not clear how to distinguish A. fumigatus-related lung damage from lung damage caused by other CF-related microorganisms. Diagnostic imaging techniques can be important to make this distinction and to aid clinical decision-making on whether to start antifungal 
treatment. The threshold to start such treatment in PwCF with positive cultures for A. fumigatus is high, as antifungal drugs can have substantial side effects. Furthermore, there are no well-conducted trials available in CF to guide treatment and anti-fungal drugs are known to have a high number of drug-drug interactions with other CF medications. Therefore, it is important to identify within the larger group of A. fumigatus-positive PwCF those individuals that require antifungal treatment to avoid accelerated progression of structural lung damage and lung function decline. For those subjects, negative consequences of antifungal treatment will likely be outweighed by the advantages, because halting or slowing down the progression of structural lung damage will have a direct effect on their life expectancy and quality of life.

For this review, we will first give an overview on how A. fumigatus colonisation and infection in PwCF can develop and discuss the different types of pulmonary disease caused by A. fumigatus. Next, we will elaborate on the role imaging can play in the detection of A. fumigatus-related structural lung damage and in the differentiation between the different types of pulmonary disease it can cause in PwCF.

\section{Background of $A$. fumigatus}

Sources of Aspergillus

Although over 150 species of Aspergillus have been described, only a few can cause disease in humans [24]. The most important Aspergillus species associated with pulmonary disease is A. fumigatus [20, 25]. Commonly, A. fumigatus grows ubiquitously in soil, on carbon-rich substrates or as a contaminant of starchy foods such as bread and potatoes (figure 1a). Furthermore, studies have shown that patients colonised with $A$. fumigatus may become the source of patient-to-patient transmission via cough aerosols or sputum [26, 27]. A. fumigatus has different stages in its life cycle with different morphological forms. Conidia, which are tiny hydrophobic particles, are easily dispersed by air and are inhaled on a daily basis by humans (figure 1b). Conidia are the infective form of the Aspergillus, while hyphae are the invasive form causing invasive disease (figure 1c, d). Conidia in the airways of PwCF can become metabolically active and secrete antigens which can provoke an allergic response. Once conidia land in a suitable environment with sufficient nutrients, such as the lungs, they can start to germinate and form hyphae which can cause an invasive infection (figure 1d) [28, 29].

\section{Inhalation}

People inhale on average between 100 and 1000 conidia per day [28, 30, 31]. The mass median aerodynamic diameter of the conidia is in the order of 2 to $3 \mu \mathrm{m}$, with extremes up to $3.5 \mu \mathrm{m}$ [31, 32].

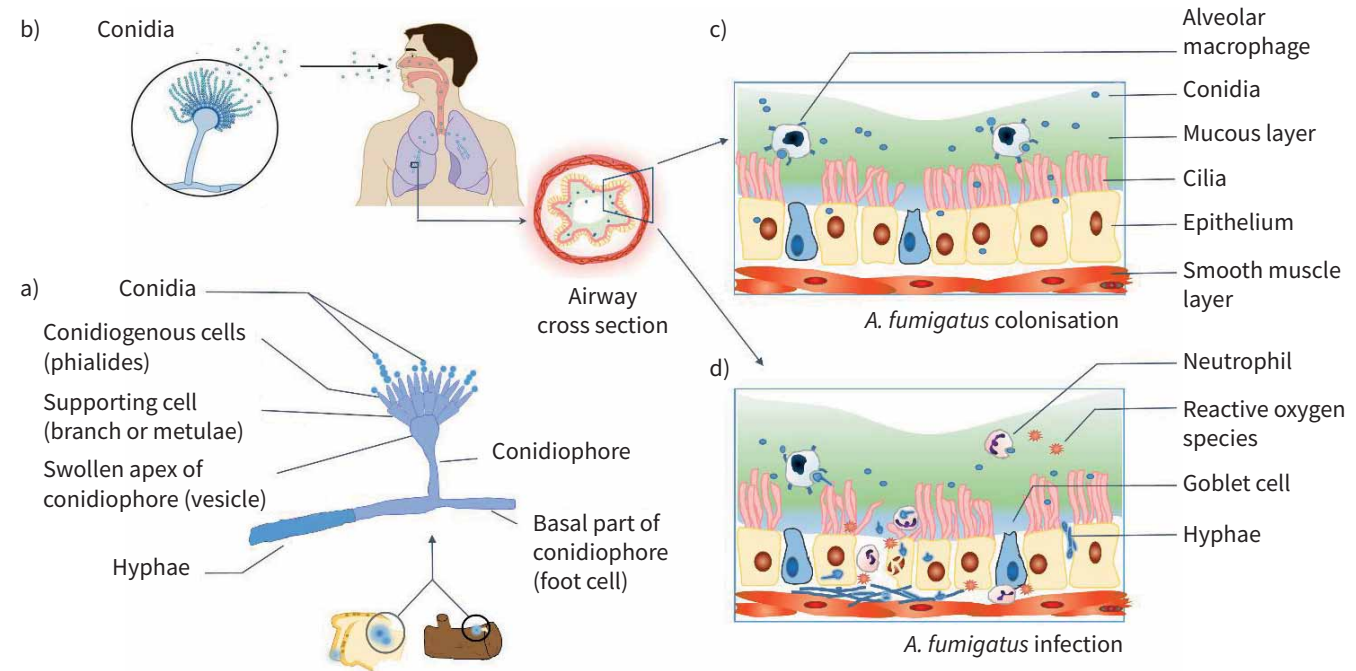

FIGURE 1 Aspergillus fumigatus from transmission to colonisation or infection. a) Microscopic morphology of Aspergillus which can grow on dead wood or starchy foods [29]. b) Inhalation of conidia into airways. Panel b) is reproduced and modified from [29] with permission. c) Colonisation of $A$. fumigatus in the airways in people with cystic fibrosis, where due to impaired mucociliary clearance of the thick mucus layer, the inhaled conidia are not eliminated. d) A. fumigatus infection in which germination of conidia leads to hyphal growth and tissue invasion triggering neutrophil recruitment and the release of reactive oxygen species which mediate a multitude of inflammatory pathways causing damage to the airways. 
Hence, conidia are in the respirable range, meaning that they are small enough to bypass the upper airways to get deposited into the peripheral airways and even into the alveoli [33-35]. In healthy subjects, mucociliary clearance will be able to clear inhaled conidia effectively. Furthermore, alveolar macrophages as the first line of defence can eliminate conidia at an early stage, thereby avoiding germination [11, 16, 36-38]. In PwCF, the mucociliary clearance is severely compromised and CFTR-defective alveolar macrophages are less effective in the elimination of conidia. Therefore, inhaled conidia have a higher chance to germinate. Furthermore, it has been shown that dysfunction of epithelial cells in CF influences the interaction with $A$. fumigatus and leads to inflammation of the lungs [16, 39].

\section{Colonisation}

Colonisation by $A$. fumigatus is defined as the presence of this fungus in laboratory cultures of the respiratory tract without clinical symptoms or deterioration in lung function (table 1 [13, 40, 41]) [16, 25, 42]. The exact prevalence of colonisation in PwCF is not well known and reports vary at between $3.4 \%$ and $57 \%[11,38,43]$.

Rates of colonisation are associated with levels of exposure [11, 44]. Certain climate factors, such as temperature, wind speed and humidity alter the load of A. fumigatus in the air [45], which may influence rates of colonisation in PwCF. For example, most reports show that higher concentrations of airborne conidia are detected in warmer weather, especially during summer and autumn, but without significant differences both in colonisation rate and airborne counts [46-49].

TABLE 1 Differences between Aspergillus fumigatus colonisation/infection and disease diagnosis and presentation in people with cystic fibrosis [13, $40,41]$

Colonisation/infection

Disease

Aspergilloma Aspergillus bronchitis/ ABPA
bronchiolitis

\begin{tabular}{|c|c|c|c|c|}
\hline \multicolumn{5}{|l|}{ Diagnostic tests } \\
\hline Fungal airway culture & + & $t^{\#}$ & + & + \\
\hline Positive RT-PCR & + & + & + & + \\
\hline \multicolumn{5}{|l|}{ Serology } \\
\hline Total IgE & $=$ & $\uparrow$ & $=/ \uparrow$ & $\uparrow \uparrow$ \\
\hline A. fumigatus-specific IgE/IgG & $=$ & $\uparrow$ & $-/ \uparrow$ & $\uparrow$ \\
\hline Galactomannan & - & $\uparrow$ & $\uparrow$ & $\uparrow$ \\
\hline \multicolumn{5}{|l|}{ Signs and symptoms } \\
\hline $\begin{array}{l}\text { Clinical symptoms (productive } \\
\text { cough, tenacious sputum, } \\
\text { breathlessness or } \\
\text { haemoptysis) }\end{array}$ & $=$ & $=/ \uparrow$ & $=/ \uparrow$ & $\uparrow \uparrow$ \\
\hline Pulmonary function & $=/ \downarrow$ & $=/ \downarrow$ & $\downarrow$ & $\downarrow$ \\
\hline \multicolumn{5}{|l|}{ Most common imaging features } \\
\hline $\mathrm{CR}$ & None/aspecific & $\begin{array}{l}\text { Spherical/ovoid } \\
\text { mass within a } \\
\text { cavity (Monod } \\
\text { sign") }\end{array}$ & $\begin{array}{c}\text { Bronchopneumonia pattern } \\
\text { with alveolar or peribronchial } \\
\text { opacity }\end{array}$ & $\begin{array}{c}\text { Transitory alveolar } \\
\text { opacities, central mucus } \\
\text { plugs (finger in glove sign) }\end{array}$ \\
\hline CT & $\begin{array}{l}\text { BWT, low attenuation } \\
\text { regions increased, some } \\
\text { mucus plugs }\end{array}$ & $\begin{array}{l}\text { Fungal ball in } \\
\text { pre-existing cavity }\end{array}$ & $\begin{array}{l}\text { Bronchiolits pattern with } \\
\text { centrilobular nodules, } \\
\text { tree-in-bud, BWT, BE, GGO, } \\
\text { peribronchial consolidation }\end{array}$ & $\begin{array}{l}\text { Central BE with mucus } \\
\text { plugs and mucus with high } \\
\text { density ( } 30 \% \text { of cases, } \\
\text { attenuation value }>70 \mathrm{HU} \text { ) }\end{array}$ \\
\hline MRI & $\begin{array}{l}\text { Low intensity regions } \\
\text { (representing trapped air } \\
\text { or hypoperfusion), T2 } \\
\text { hyperintense plugs }\end{array}$ & $\begin{array}{l}\text { Monod sign": } \\
\text { fungal ball with } \\
\text { low T1WI and } \\
\text { T2WI signal }\end{array}$ & $\begin{array}{c}\text { BWT, T2WI hyperintense } \\
\text { peribronchial consolidation } \\
\text { and peripheral mucus plugs } \\
\text { (tree-in-bud }{ }^{+} \text {) }\end{array}$ & $\begin{array}{l}\text { BWT and T2 hyperintense } \\
\text { mucus plugs (in } 30 \% \text { of } \\
\text { cases inverted mucus sign, } \\
\text { mucus with high T1WI and } \\
\text { low T2WI signal) }\end{array}$ \\
\hline
\end{tabular}


Risk factors for A. fumigatus colonisation include previous treatment with steroids or antibiotics. Short courses of steroids in PwCF have been associated with an increased risk of positive sputum cultures for A. fumigatus [11, 13, 20, 25, 34, 50-55]. Furthermore, long-term antibiotic exposure may also provide a more favourable environment for fungi to grow, causing $A$. fumigatus colonisation to occur $[6,54,55]$.

\section{Interactions}

Another risk factor for persistent colonisation with A. fumigatus is co-infection with $P$. aeruginosa. There is a lot of conflicting literature about the interaction of co-infection between A. fumigatus and $P$. aeruginosa. The prevalence of chronic $P$. aeruginosa infection currently ranges from $3.3 \%$ to $26 \%$ for children and between $41 \%$ and $52 \%$ for adults with CF [56]. In vitro research has shown that the production of cytotoxic elastase by $P$. aeruginosa increases in co-culture with A. fumigatus, leading to damage to the human lung epithelial cells $[54,57]$. Alternatively, eradication treatment for $P$. aeruginosa could create a microbial environment favourable for A. fumigatus colonisation [58]. Data from the Irish CF registry showed that co-colonisation with $P$. aeruginosa and A. fumigatus was associated with a $165 \%$ increase in hospital admissions, a $112 \%$ increase in the number of respiratory exacerbations and a $48 \%$ increase in the number of oral antimicrobial courses, compared to patients with negative cultures for both pathogens [59].

Apart from P. aeruginosa, interactions between A. fumigatus and other bacterial infections, such as Staphylococcus aureus, Streptococcus pneumoniae, Klebsiella pneumoniae and Stenotrophomonas maltophilia, do not only lead to superinfection but also increase the chance of A. fumigatus infection evolving into pulmonary disease in PwCF [60]. In addition, in PwCF, a positive fungal culture is often associated with a positive culture for nontuberculous mycobacteria [61, 62], a combination that seems to predispose to the development of chronic pulmonary aspergillosis [63, 64]. Therefore, further research is needed on these complex interactions between microorganisms and their effect on clinical outcomes in PwCF.

Recently, the interaction between anti-fungal treatment and CFTR modulators has attracted attention in PwCF, as the treatment of chronic Aspergillus infection has become troublesome for those who are also receiving modulator therapy. CFTR modulators, which can enhance the functional expression of specific CFTR mutations, also impact fungal colonisation [65]. On one hand, CFTR modulator studies have shown ivacaftor, lumacaftor and tezacaftor to have a positive effect on the dysregulated host response to Aspergillus in PwCF [66], which may reduce airway inflammation from Aspergillus infection. On the other hand, CFTR modulators can have drug-drug interactions with antifungal medication. For instance, the cytochrome P450-3A-inducing effect of lumacaftor can make it difficult to reach clinically effective blood levels of orally administered antifungal medications [65].

\section{Infection}

A. fumigatus infection occurs when the conidia in the airway trigger the release of harmful factors such as antigenic proteins and toxins, and/or germinate into hyphae becoming invasive (figure 1d), thereby initiating an inflammatory response. Neutrophils are recruited resulting in high levels of reactive oxygen species which mediate a multitude of inflammatory pathways causing even more damage to the airways $[67,68]$. Furthermore, A. fumigatus produces some secondary metabolites, such as proteolytic enzymes and gliotoxins, leading to epithelial cell detachment and further impaired mucociliary clearance and enhanced inflammation [6, 18, 34, 35, 38]. A mycobiome analysis approach in a non-CF bronchiectasis cohort has been reported, which stated that the pulmonary mycobiome, in which A. fumigatus is one of the dominated profiles, is of clinical relevance in the development of bronchiectasis [23]. The multi-omics approach will likely be a benefit for diagnosing Aspergillus infection, as the characterisation of the hostfungal interaction and the use of multi-omics strategies may lead to a better understanding of host biology and its interaction with fungal pathogens [69].

In PwCF, pulmonary aspergillosis can be subclassified in three distinct clinical manifestations: aspergilloma, Aspergillus bronchitis/bronchiolitis and allergic infection. Insight is lacking why some PwCF do not develop Aspergillus disease upon exposure, while others develop hyper-inflammatory disease characterised by a Th1-driven inflammation or Th2-driven allergic hyper-inflammation. Over time, PwCF can present with different Aspergillus disease presentations and move between one entity and the other, which is an observation not yet explained.

\section{Pulmonary aspergilloma}

An aspergilloma is the development of a fungal ball in a pre-existing cavitary lesion or ectatic bronchus without signs of tissue invasion [70, 71]. Complex aspergilloma is nowadays referred to as chronic cavitary pulmonary aspergillosis, indicating multiple pulmonary cavities containing one or more 
aspergillomas [72, 73]. In PwCF, this is a rare complication that can present in those patients colonised with A. fumigatus and severe or end-stage pulmonary disease [74, 75], and in patients with large cystic bronchiectasis. Aspergilloma can be discovered on computed tomography (CT) as an accidental finding or after an episode of haemoptysis without any specific other symptoms [37, 76].

\section{Aspergillus bronchitis/bronchiolitis}

Invasive pulmonary aspergillosis (IPA) is defined as a condition where the hyphae invade the bronchial wall or adjacent lung parenchyma, such as the pulmonary vasculature $[77,78]$. IPA is only seen in the case of immunodeficiency and as a rare entity in CF patients. In PwCF, Aspergillus bronchitis/bronchiolitis is the more common infection type. Aspergillus bronchitis, first reported in 2006 in a cohort of six CF patients [79], is defined as a chronic superficial infection of the airways [80]. The reported prevalence of Aspergillus bronchitis is 9\% in PwCF, but likely to be under-reported [81]. Aspergillus bronchitis is defined as: a) symptoms related to chronic lower airway disease; b) Aspergillus species detected in sputum or BAL by culture or real-time polymerase chain reaction test; and c) Aspergillus-specific IgG in serum [80]. Clinical symptoms associated with Aspergillus bronchitis are productive cough, tenacious sputum, breathlessness and haemoptysis and can hardly be distinguished from a bacterial exacerbation in PwCF $[40,80]$.

Allergic pulmonary aspergillosis infection (ABPA)

ABPA is a serious complication in PwCF and the result of a complex immunologic response against A. fumigatus. ABPA has a prevalence of $4-9 \%$ in children and around $10 \%$ in adult PwCF [11, 82-88]. ABPA individuals sensitised to A. fumigatus can develop an IgE-mediated allergic inflammatory reaction (type I immediate hypersensitivity) which leads to an acute inflammatory response resulting in mucus impaction and bronchial obstruction after inhaling $A$. fumigatus spores [89-91]. ABPA mice models show that chronic exposure to A. fumigatus antigens may lead to fibrosis and irreversible lung damage [10, 89].

The diagnosis of ABPA can be difficult in PwCF because of atypical clinical symptoms such as cough, wheeze and increased sputum production [44, 92, 93]. Patients typically show a drop in forced expiratory volume in the first second that does not respond to regular antibiotic treatment directed against bacteria cultured from their sputum. The diagnosis can be supported by typical immunologic features such as an elevated total IgE concentration (greater than $1000 \mathrm{ng} \cdot \mathrm{mL}^{-1}$ or $416 \mathrm{IU} \cdot \mathrm{mL}^{-1}$ ), increased Aspergillus specific IgE antibodies (greater than 1.91 kilo units of antibody per litre), or increased Aspergillus specific IgG antibodies (greater than $78 \mathrm{mg} \cdot \mathrm{L}^{-1}$ ) $[25,37,40,89,94-100]$. The basophil activation test, an in vitro test to measure the activation of basophils by IgE stimulation with A. fumigatus extract and CD63, CD193 and CD203c [101], can be used to discriminate ABPA from Aspergillus colonisation and non-ABPA in PwCF [102]. Furthermore, the results of basophil activation tests are not affected by antifungal or corticosteroid treatment; therefore, this test might be used as an additional criterion to identify ABPA and monitor A. fumigatus-related clinical status in PwCF [102-104].

\section{Imaging and aspergillosis}

Despite the currently available diagnostic tools, it remains challenging to differentiate colonisation versus infection with Aspergillus according to clinical tests (table 1). In clinical practice, it is also difficult to distinguish progression of CF lung disease due to Aspergillus from progression related to other, mainly bacterial, airway pathogens. Therefore, an important goal for clinicians is to find the particular fingerprint for Aspergillus-related lung damage (figure 2).

Imaging is an essential tool in the management of patients with pulmonary disease related to $A$. fumigatus $[105,106]$. The two most used imaging tools to screen patients at high risk for pulmonary aspergillosis are chest radiography (CR) and CT. Both techniques are used to detect pulmonary disease related to A. fumigatus in patients with compatible illnesses, differentiate aspergillosis from other diseases, guide interventional procedures aimed at establishing a specific diagnosis and assess treatment response. CT is much more sensitive and specific than CR [107] in the evaluation of chest lesions and therefore considered the gold standard for an accurate differential diagnosis of different forms of pulmonary aspergillosis. CR, however, because of its high accessibility, low cost and radiation exposure to the patient, is frequently used when high frequency repetitive or bedside imaging of critically ill patients is required. More recently, magnetic resonance imaging (MRI) has been proposed as an imaging tool to diagnose specific subtypes of pulmonary aspergillosis [108-111]. A main advantage of MRI over CT is the ability to follow-up response to treatment with serial imaging without concerns related to radiation exposure. MRI has been increasingly used in those tertiary centres with experience in chest MRI. 


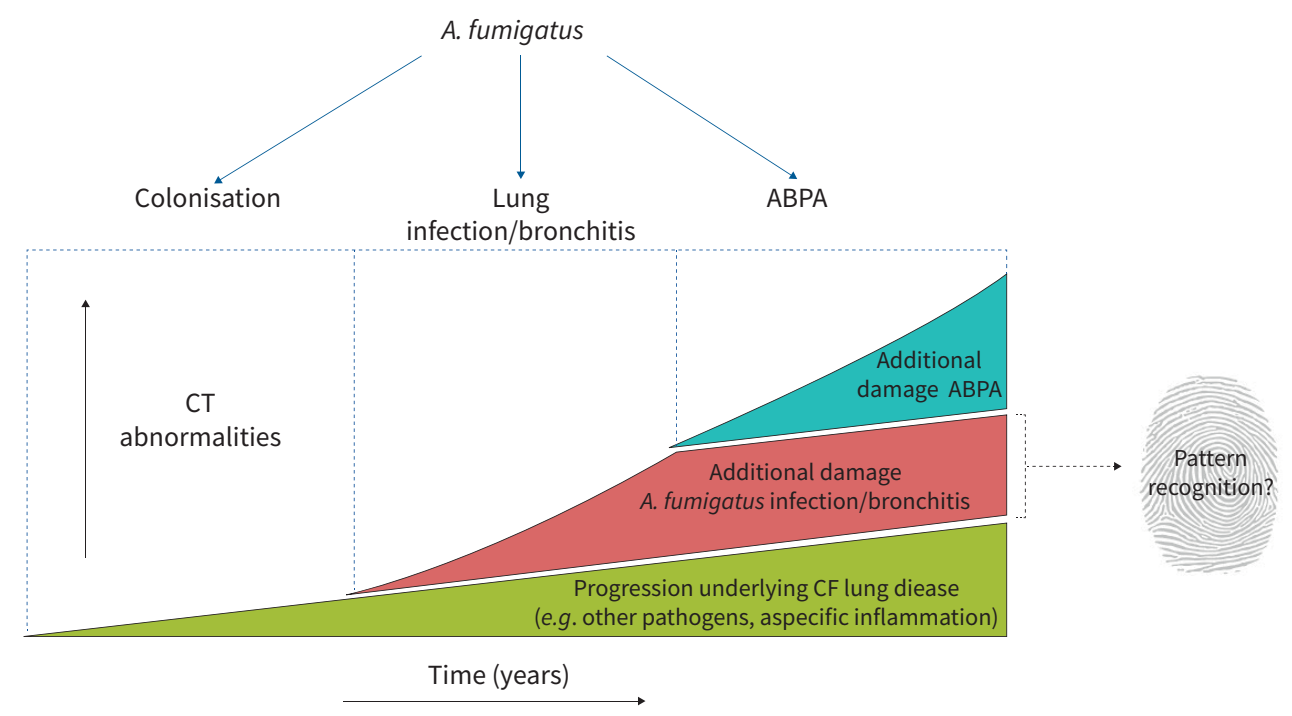

FIGURE 2 The distinction between cystic fibrosis (CF)-related and Aspergillus fumigatus-related structural lung damage in people with CF. The figure represents the difficulty of distinguishing "normal" progression of CF-related lung disease on chest computed tomography (CT) from A. fumigatus infection/bronchitis-related progression and allergic bronchopulmonary aspergillosis (ABPA)-related structural lung disease on top of this progression, and our goal to find the fingerprint for Aspergillus-related lung damage. Note: ABPA and Aspergillus bronchitis can either co-exist or occur separately.

In the section below we will discuss the most common radiological hallmarks of $A$. fumigatus colonisation, Aspergillus bronchitis and ABPA using CT and MRI in PwCF. Imaging features of each subtype of Aspergillus infection in PwCF are summarised in table 1.

\section{Imaging features of A. fumigatus colonisation}

In theory, one would expect that mere colonisation with A. fumigatus would not be related to any identifiable abnormalities on CT. In contrast, the few imaging studies that have looked into the relationship between A. fumigatus colonisation and structural lung changes in CF did report significant differences [2, 58]. In particular, PwCF who are colonised with A. fumigatus were found to have more low attenuation regions (often referred to as "trapped air") on expiratory chest CT than a control group without colonisation [58]. There are also indications that this process can start early in life. In a study of a 5-year-old PwCF, the presence of $A$. fumigatus in the BAL fluid was associated with low attenuation regions on chest CT [58]. In addition, more severe bronchiectasis and more mucus plugging were observed in a group of A. fumigatus colonised PwCF compared to a non-colonised group [2]. Causal associations between A. fumigatus and the reported structural lung abnormalities cannot be asserted from these observational studies, but they do strongly suggest that airway cultures positive for A. fumigatus may not be an innocent finding in asymptomatic patients. Hence, at least a proportion of patients who are considered to be merely colonised by A. fumigatus according to current standards might in fact have an A. fumigatus infection.

\section{Imaging features of saprophytic Aspergillus infection}

Saprophytic aspergillosis or aspergilloma can be identified on chest CT as a fungal ball in a pre-existing pulmonary cavity, cyst or other air-containing space [97, 112]. Single or multiply lesions of aspergilloma can affect both lungs with equal frequency [113]. The mean diameter of aspergillomas is around 4 to $5 \mathrm{~cm}$, with a maximum size of around $10 \mathrm{~cm}$, and rarely shows regression in size without treatment [113].

An early CT finding of aspergilloma in the non-CF population and PwCF is the thickening of the lateral cavity wall, which reflects the saprophytic germination of the fungus onto the cavity wall. When this germination is large enough it will fall from the wall and form the aspergilloma (figure 3). The position of the fungal ball in the cavity on CT may change depending on the posture of the subject [78]. Pleural thickening can also be found on CT in association with A. fumigatus infection, which may present as early as 2-3 years before the formation of the fungal ball [78, 114, 115]. On MRI, the characteristic of aspergilloma shows low signal intensity on T1- and variable signal on T2-weighted images caused by 

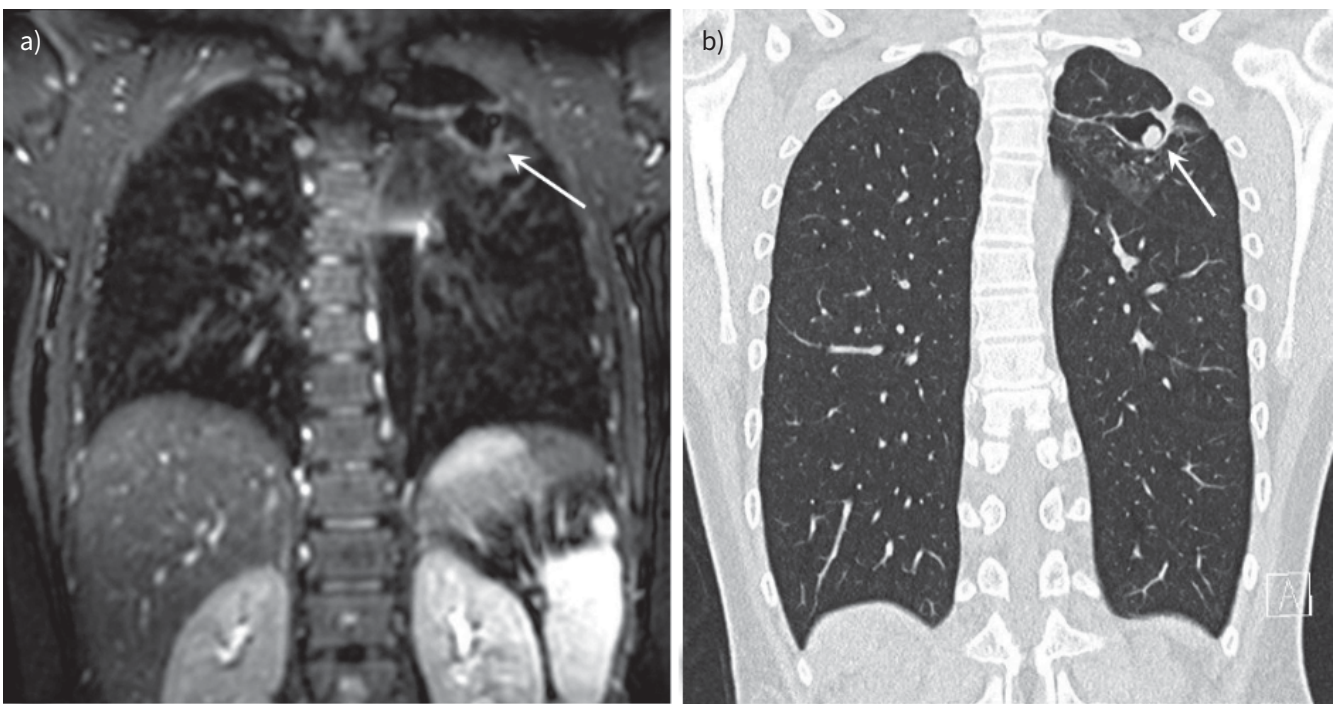

FIGURE 3 Chest imaging of a 13 -year-old boy with cystic fibrosis. a) Magnetic resonance imaging (MRI) scan (isotropic T2-weighted CUBE, coronal reformat) and b) computed tomography (CT) scan (isotropic coronal reformat) performed 10 months after the MRI. Note cavitary lesion in the left upper lobe on MRI (arrow) with wall thickening but without a solid component. On the follow-up CT, there is a new round solid lesion within the cavity, representing an aspergilloma (arrow) not completely filling the cavity (Modod sign).

calcium, air or ferromagnetic elements in the aspergilloma [116]. Air surrounding a fungal ball that does not completely fill up the cavity can be recognised as the Monod sign (figure 3) [78].

Imaging features of Aspergillus bronchitis/bronchiolitis

Histology of patients diagnosed as having Aspergillus bronchitis show superficial invasion by A. fumigatus of the superficial mucosal layers [21]. Therefore, CT findings in Aspergillus bronchitis follow the bronchocentric invasion of the airways. The initial signs of infection are bronchial wall thickening and centric-lobular nodules with ground glass opacities (GGOs) (figure 4a) with development of peri-bronchiolar and lobar consolidations as the infection progresses with consequent filling of airspaces (figure 4b). However, these findings can be non-specific, especially in PwCF [77, 78, 117]. The same holds for bronchiectasis, which has been described on CT in Aspergillus bronchitis in patients with non-CF chronic lung disorders, but is non-specific in PwCF [117]. To our knowledge there is only one case report on Aspergillus bronchitis in CF with a detailed description of CT findings, reporting signs of bronchitis/bronchiolitis with centrilobular nodules, tree-in-bud, GGO and peribronchial consolidations [118].

\section{Allergic pulmonary aspergillosis}

The most common radiological sign in ABPA is central varicose or cystic bronchiectasis in both non-CF patients and PwCF [10, 84, 91, 98, 119], also described as a "string of pearls" [120]. Another imaging sign of ABPA is mucous impaction in the central airways [99]. Furthermore, high attenuation regions on CT, with a mucus density higher than the para-spinal skeletal muscle (generally $>70$ Hounsfield units), are considered a specific feature for the radiological diagnosis of ABPA in CF [41, 84, 86, 121-125] (figure $5 \mathrm{a}, \mathrm{b})$. Nodule or granuloma formation can be detected in the periphery of the lungs [85], as well as large opacities, which tend to affect the upper and middle lobes [90, 126]. In addition, improvement of the imaging abnormalities in response to steroid treatment supports the diagnosis of ABPA in PwCF [89, 126].

General features of CF lung disease and early changes related to ABPA are difficult to differentiate. For the diagnosis and follow-up of ABPA, MRI has advantages over CT as it does not require ionising radiation. Furthermore, MRI allows the collection of morphological and functional information that might allow a more sensitive and specific diagnosis of ABPA-related changes [116]. Some studies with a small sample size have reported MRI findings of ABPA, especially in paediatric patients [108, 109]. These studies indicate that high T1 and low T2 signal intensities of mucus impaction (inverted impaction mucus sign) are both sensitive and specific for the diagnosis of ABPA in CF (figure 5c, d) [108-111]. This high discriminating ability of ABPA by MRI requires further confirmation in a larger number of PwCF. 

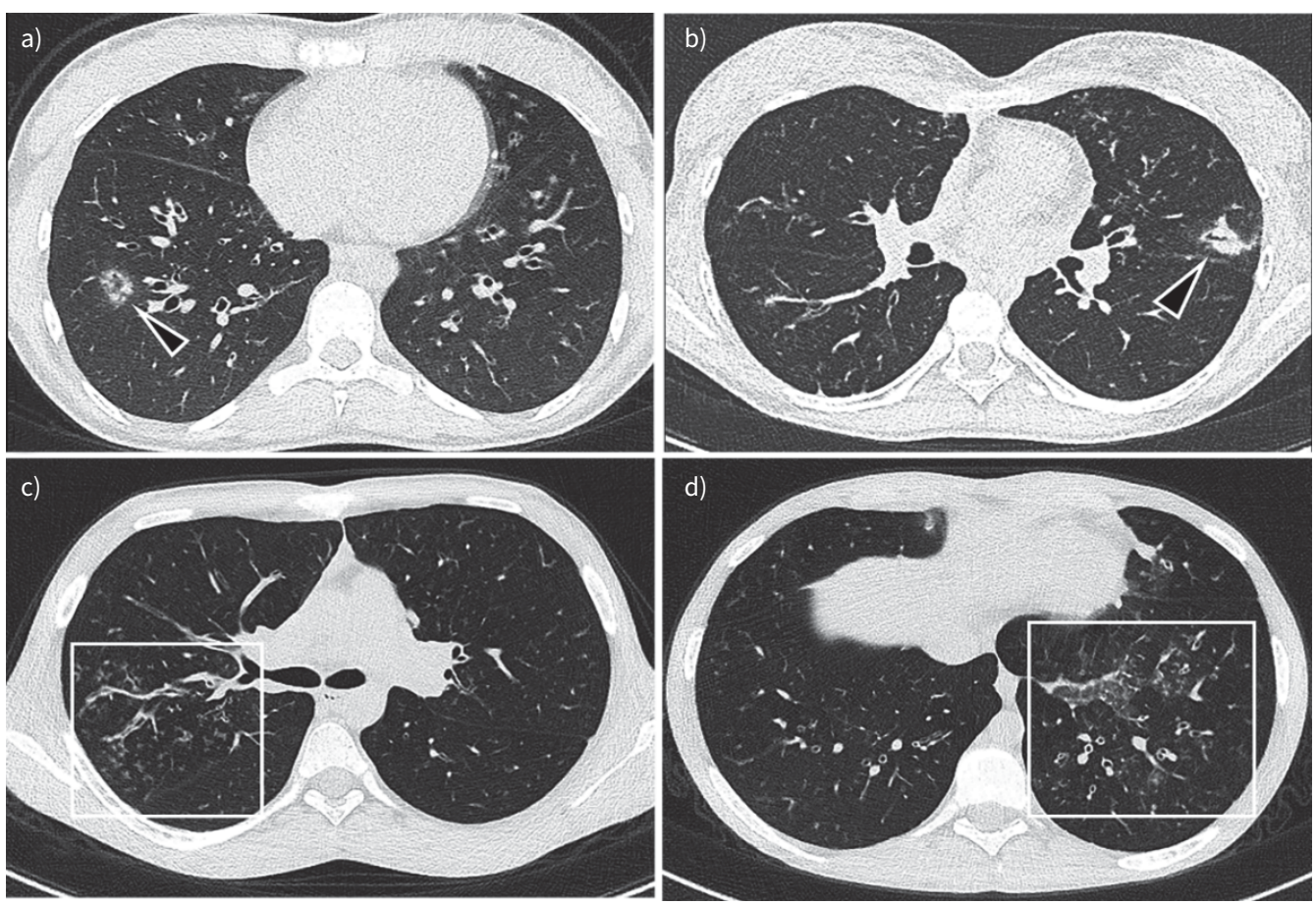

FIGURE 4 Aspergillus bronchitis/bronchiolitis example images: a) 18-year-old male non-cystic fibrosis (CF) patient, but with sickle cell disease. Multiple nodules with ground glass opacity around peripheral vessels are shown in the right lower lobe (arrowhead in a). b) Chest computed tomography (CT) image of a 17-year-old girl with CF shows Aspergillus bronchopneumonia. Note bronchocentric peri-bronchiolar consolidation on left upper lobe; $\mathrm{C}$ and d) Chest CT images of a 15-year-old boy with CF show Aspergillus bronchiolitis. Note filling of peripheral airways with a tree-in-bud pattern and bronchial wall thickening (box in c). In $d$ there is also an area of ground glass (box in d).

Without antifungal treatment, Aspergillus diseases may move to chronic infections with complex appearances on image exams. The granulomas presenting in chronic Aspergillus infection indicate limited tissue invasion, which is often accompanied by enlarged intra-pulmonary lymph nodes (figure 6). On CT imaging, Aspergillus granulomas may cause extensive parenchymal consolidations, may lead to bronchiectatic cavities or may remain exclusively bronchogenic. The latter pattern is characterised by multiple nodules with a perilymphatic distribution (subpleural and perivisceral). Adjacent pleura can be invaded as well, which leads to the formation of cavitations, aspergilloma and pleural thickening on CT [73].

\section{The way forward}

Based on what is known in the literature, there are strong indications that $A$. fumigatus can contribute to the progression of structural lung damage in PwCF. As A. fumigatus can be cultured in a high percentage of PwCF, it is important to differentiate colonisation versus infection and to develop state-of-the-art sensitive and specific image analysis systems that are able to identify A. fumigatus-associated structural abnormalities on chest CT and/or MRI. This will support differentiation between harmless colonisation and infection with its associated structural lung damage. Such an image analysis system can be used for PwCF and A. fumigatus-positive sputum cultures to assist in the early detection and monitoring of A. fumigatus-associated structural lung damage and to monitor the effect of antifungal treatment.

Routine biennial chest CTs are used in around half of the European CF Society (ECFS) clinical trial network (CTN) centres. All these ECFS-CTN centres contribute data to the ECFS patient registry. The availability of an automated image analysis system sensitive and specific to detect A. fumigatus-associated structural changes opens up the possibility to analyse a large number of chest CTs to add key imaging outcomes to the registry. This will allow a better understanding of A. fumigatus induced lung damage. In addition, such a system can play an important role as outcome measures for clinical studies. An example of this is the recently started international cASPerCF study (clinicaltrial.gov identifier NTC 01782131) [127] evaluating the therapeutic effect of posaconazole treatment in children and adolescents with CF and 

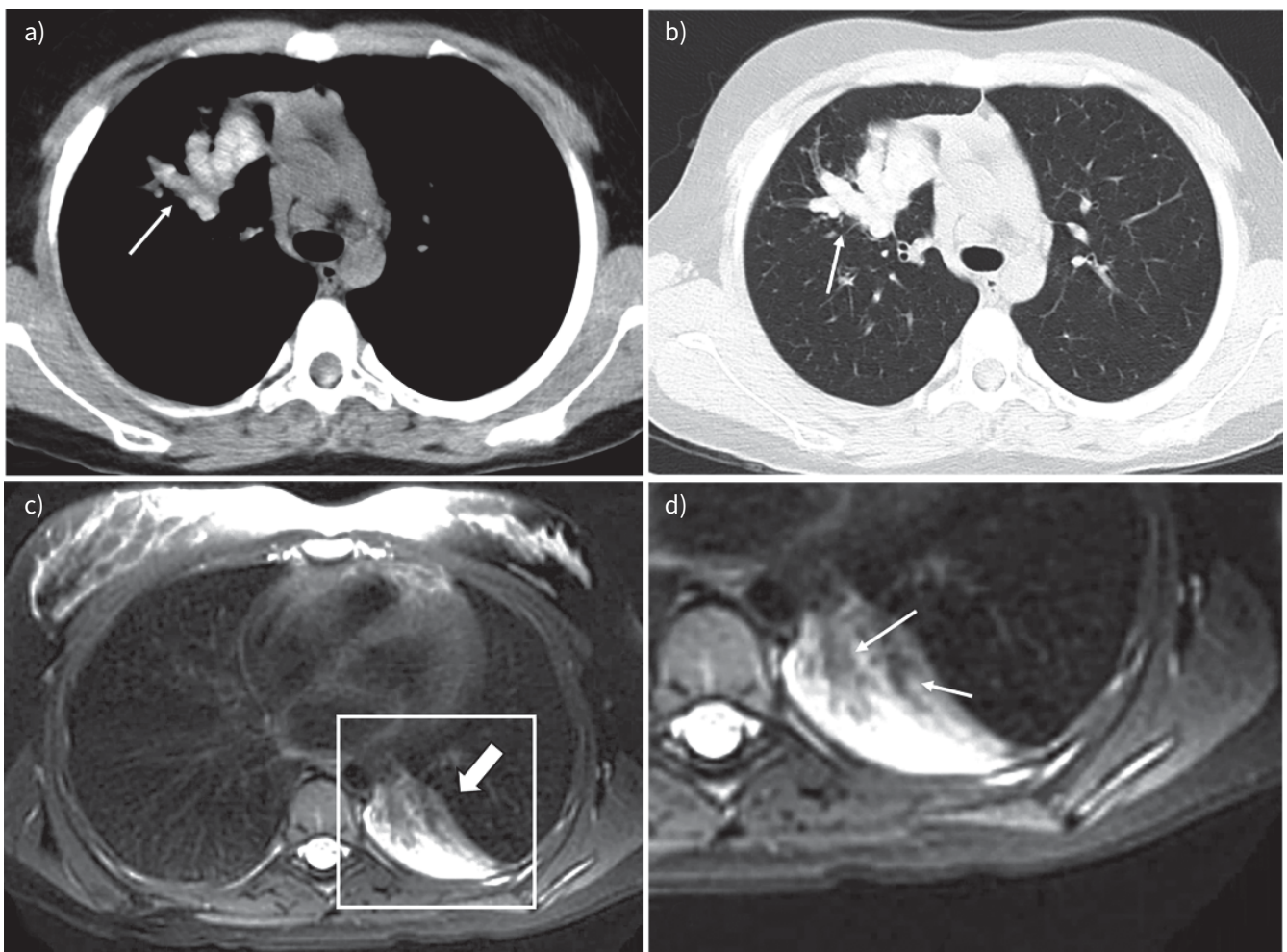

FIGURE 5 Allergic bronchopulmonary aspergillosis example images. a) and b) Chest computed tomography (CT) images of a 12-year-old boy with cystic fibrosis (CF). a) $2.5 \mathrm{~mm}$ axial CT soft tissue window and b) $1 \mathrm{~mm}$ axial CT lung window at the same level. Note in a), central bronchiectasis filled with high density material (arrow). In b), note mucus plugs with a typical cylindrical shape originating from the hilar region and extending towards the periphery (arrow) showing typical higher density than paraspinal muscles. c) and d) Lung magnetic resonance imaging scans (T2-weighted axial PROPELLER, in plane resolution $1 \times 1 \mathrm{~mm}$, slice thickness $5 \mathrm{~mm}$ ) of a 15-year-old girl with CF. c) Atelectasis in the left lower lobe (thick arrow). d) Hypo-intense material filling the central airways (arrows).

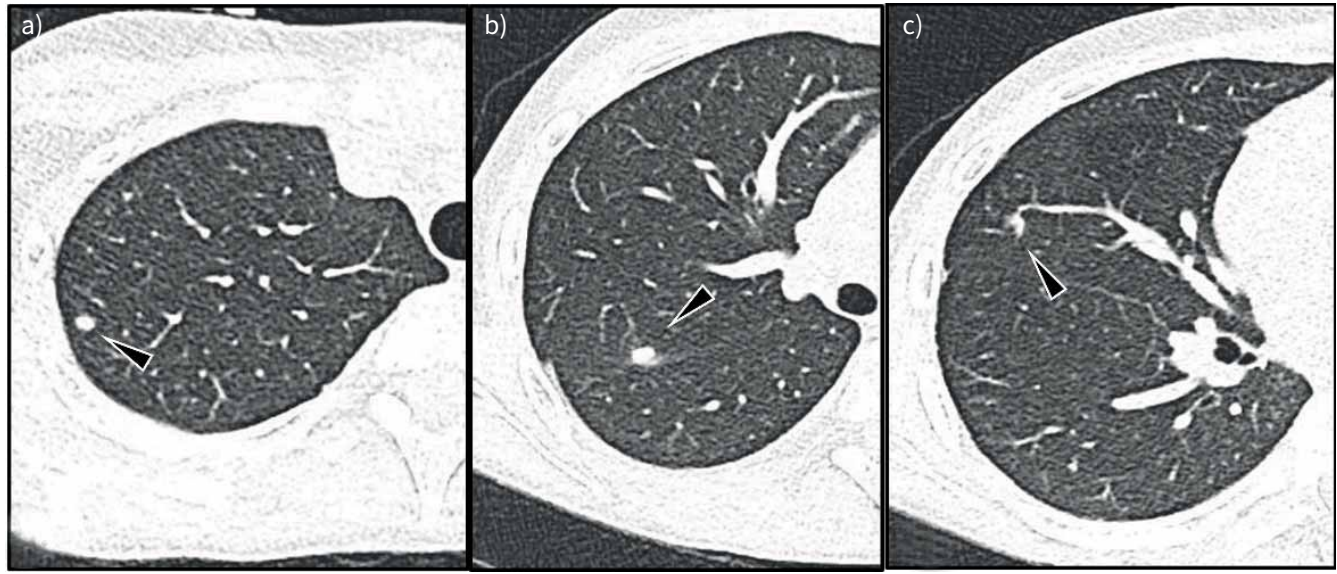

FIGURE 6 Chronic aspergillosis with skin and lung abnormality in a 15-year-old boy with cardiomyopathy. Note sub-pleural (arrowhead in a) and peri-fissural (arrowheads in b and c) nodules. From their location, these nodules are most likely intrapulmonary lymph nodes, which disappeared after 4 months of treatment with antifungal medication (voriconazole). 
A. fumigatus infection. The cASPerCF study will explore whether chest CT-related outcomes are sensitive to detect a therapeutic effect.

Furthermore, chest MRI could also play an important role in the differential diagnosis and follow-up of pulmonary aspergillosis. Chest MRI could be used as an imaging tool for short-term follow-up of therapy changes in PwCF. In our centre, we are currently using a short scanning protocol including T2-weighted and proton density-weighted imaging to follow treatment changes in PwCF and Aspergillus infection, namely consolidation and large mucus plugs. The complementary role of chest MRI in pulmonary aspergillosis will become clinically relevant when this technique has broader use in clinical practice. Current limitations to the use of MRI in clinical practice are the lack of protocol standardisation between CF centres and major differences in image quality, which hampers its applicability on a large scale. Finally, post-processing tools for chest MRI are not commercially available and most of the time are reported in the literature as in-house developed tools.

\section{Conclusion}

Our review provides support that specific structural lung damage on chest CT associated with A. fumigatus infection in PwCF can be identified. The development of sensitive and specific image analysis methods for chest CT and MRI are needed to identify whether in PwCF A. fumigatus-related structural lung damage can be detected at an early stage to guide treatment. Furthermore, image-related outcomes can be important as outcome measures for clinical studies and for clinical management.

Provenance: Submitted article, peer reviewed.

Acknowledgements: We would like to acknowledge W.M. Bramer (Erasmus MC medical library) for his assistance in the literature search. We would like to thank T. van der Veer (Erasmus MC) for providing figure 3.

Author contributions: Q. Lv contributed to the design of the work, drafting the manuscript, drawing the figure, and gave final approval to the manuscript. B.B.L.J. Elders contributed to critically revising the manuscript and gave final approval to the manuscript. A. Warris contributed to critically revising and gave final approval to the manuscript. D. Caudri contributed to revising the manuscript, drawing the figure, and gave final approval to the manuscript. P. Ciet contributed to the design of the work, picture selections and gave final approval to the manuscript. H. Tiddens contributed to the design of the work, critically revised the manuscript, and gave final approval to the manuscript.

Conflict of interest: Q. Lv has nothing to disclose. B.B.L.J. Elders has nothing to disclose. A. Warris has nothing to disclose. D. Caudri has nothing to disclose. P. Ciet reports personal fees from Vertex Pharmaceutical, outside the submitted work. H. Tiddens reports grants and other funding from Novartis, grants from CFF and Vectura, and personal fees from Vertex, Thirona and Insmed, outside the submitted work. In addition, Erasmus MC and Telethon Kids Institute have licensed the use of PRAGMA-CF to Thirona and Resonance Health. The Sophia research BV of the Erasmus MC-Sophia Childrens hospital has received unconditional research grants from Novartis, and Vectura.

Support statement: Funding was received from Nederlandse Cystic Fibrosis Stichting (NCFS)-Health Holland (PPS). Funding information for this article has been deposited with the Crossref Funder Registry.

\section{References}

$1 \quad$ O'Sullivan BP, Freedman SD. Cystic fibrosis. Lancet 2009; 373: 1891-1904.

2 McMahon MA, Chotirmall SH, McCullagh B, et al. Radiological abnormalities associated with Aspergillus colonization in a cystic fibrosis population. Eur J Radiol 2012; 81: e197-e202.

$3 \quad$ Ratjen F, Doring G. Cystic fibrosis. Lancet 2003; 361: 681-689.

4 Foster K, Alton H. Chronic lung infection in children. Paediatr Respir Rev 2003; 4: 225-229.

$5 \quad$ King J, Brunel SF, Warris A. Aspergillus infections in cystic fibrosis. J Infect 2016; 72: Suppl., S50-S55.

6 Pihet M, Carrere J, Cimon B, et al. Occurrence and relevance of filamentous fungi in respiratory secretions of patients with cystic fibrosis-a review. Med Mycol 2009; 47: 387-397.

7 Zemanick ET, Hoffman LR. Cystic fibrosis: microbiology and host response. Pediatr Clin North Am 2016; 63 : 617-636.

8 Richardson M, Bowyer P, Sabino R. The human lung and Aspergillus: You are what you breathe in? Med Mycol 2019; 57: Suppl. 2, S145-S154.

9 Moskowitz SM, Gibson RL, Effmann EL. Cystic fibrosis lung disease: Genetic influences, microbial interactions, and radiological assessment. Pediatr Radiol 2005; 35: 739-757. 
Moss RB. Fungi in cystic fibrosis and non-cystic fibrosis bronchiectasis. Semin Respir Crit Care Med 2015; 36 207-216.

Burgel PR, Paugam A, Hubert D, et al. Aspergillus fumigatus in the cystic fibrosis lung: Pros and cons of azole therapy. Infect Drug Resist 2016; 9: 229-238.

Saunders RV, Modha DE, Claydon A, et al. Chronic Aspergillus fumigatus colonization of the pediatric cystic fibrosis airway is common and may be associated with a more rapid decline in lung function. Med Mycol 2016; 54: 537-543.

Liu JC, Modha DE, Gaillard EA. What is the clinical significance of filamentous fungi positive sputum cultures in patients with cystic fibrosis? J Cyst Fibros 2013; 12: 187-193.

Breuer O, Schultz A, Turkovic L, et al. Changing prevalence of lower airway infections in young children with cystic fibrosis. Am J Respir Crit Care Med 2019; 200: 590-599.

Breuer O, Schultz A, Garratt LW, et al. Aspergillus infections and progression of structural lung disease in children with cystic fibrosis. Am J Respir Crit Care Med 2020; 201: 688-696.

Speirs JJ, Van Der Ent CK, Beekman JM. Effects of Aspergillus fumigatus colonization on lung function in cystic fibrosis. Curr Opin Pulm Med 2012; 18: 632-638.

Noni M, Katelari A, Dimopoulos G, et al. Aspergillus fumigatus chronic colonization and lung function decline in cystic fibrosis may have a two-way relationship. Eur J Clin Microbiol Infect Dis 2015; 34: 2235-2241.

Tomee JF, Wierenga AT, Hiemstra PS, et al. Proteases from Aspergillus fumigatus induce release of proinflammatory cytokines and cell detachment in airway epithelial cell lines. J Infect Dis 1997; 176: 300-303.

Amitani R, Taylor G, Elezis EN, et al. Purification and characterization of factors produced by Aspergillus fumigatus which affect human ciliated respiratory epithelium. Infect Immun 1995; 63: 3266-3271.

Ziesing S, Suerbaum S, Sedlacek L. Fungal epidemiology and diversity in cystic fibrosis patients over a 5-year period in a national reference center. Med Mycol 2016; 54: 781-786.

De Soyza A, Aliberti S. Bronchiectasis and Aspergillus: how are they linked? Med Mycol 2017; 55: 69-81.

Hong G, Alby K, Ng SCW, et al. The presence of Aspergillus fumigatus is associated with worse respiratory quality of life in cystic fibrosis. J Cyst Fibros 2019; 19: 125-130.

Mac Aogáin M, Chandrasekaran R, Lim AYH, et al. Immunological corollary of the pulmonary mycobiome in bronchiectasis: the CAMEB study. Eur Respir J 2018; 52: 1800766.

Denning DW. Chronic forms of pulmonary aspergillosis review. Clin Microbiol Infect 2001; 7: Suppl. 2, 25-31.

Walicka-Serzysko K, Sands D. The clinical presentations of pulmonary aspergillosis in children with cystic fibrosis-preliminary report. Dev Period Med 2015; 19: 66-79.

Lemaire B, Normand AC, Forel JM, et al. Hospitalized patient as source of Aspergillus fumigatus, 2015. Emerg Infect Dis 2018; 24: 1524-1527.

Engel TGP, Erren E, Vanden Driessche KSJ, et al. Aerosol transmission of Aspergillus fumigatus in cystic fibrosis patients in the Netherlands. Emerg Infect Dis 2019; 25: 797-799.

van de Veerdonk FL, Gresnigt MS, Romani L, et al. Aspergillus fumigatus morphology and dynamic host interactions. Nat Rev Microbiol 2017; 15: 661-674.

Latge JP, Chamilos G. Aspergillus fumigatus and aspergillosis in 2019. Clin Microbiol Rev 2019; 33: e00140-18.

Latge JP. Aspergillus fumigatus and aspergillosis. Clin Microbiol Rev 1999; 12: 310-350.

Kwon-Chung KJ, Sugui JA. Aspergillus fumigatus-what makes the species a ubiquitous human fungal pathogen? PLoS Pathog 2013; 9: e1003743.

Afanou KA, Straumfors A, Skogstad A, et al. Profile and morphology of fungal aerosols characterized by field emission scanning electron microscopy (FESEM). Aerosol Sci Technol 2015; 49: 423-435.

Tellier R, Li Y, Cowling BJ, et al. Recognition of aerosol transmission of infectious agents: a commentary. BMC Infect Dis 2019; 19: 101.

Chotirmall SH, McElvaney NG. Fungi in the cystic fibrosis lung: bystanders or pathogens? Int $J$ Biochem Cell Biol 2014; 52: 161-173.

Cowley AC, Thornton DJ, Denning DW, et al. Aspergillosis and the role of mucins in cystic fibrosis. Pediatr Pulmonol 2017; 52: 548-555.

Waldorf AR. Pulmonary defense mechanisms against opportunistic fungal pathogens. Immunol Ser 1989; 47: 243-271.

Kousha M, Tadi R, Soubani AO. Pulmonary aspergillosis: a clinical review. Eur Respir Rev 2011; 20: 156-174. Warris A, Bercusson A, Armstrong-James D. Aspergillus colonization and antifungal immunity in cystic fibrosis patients. Med Mycol 2019; 57: Suppl. 2, S118-S126.

39 Chaudhary N, Datta K, Askin FB, et al. Cystic fibrosis transmembrane conductance regulator regulates epithelial cell response to Aspergillus and resultant pulmonary inflammation. Am J Respir Crit Care Med 2012; 185: 301-310.

40 Baxter CG, Dunn G, Jones AM, et al. Novel immunologic classification of aspergillosis in adult cystic fibrosis. J Allergy Clin Immunol 2013; 132: 560-566.e10. 
41 Agarwal R, Sehgal IS, Dhooria S, et al. Radiologic criteria for the diagnosis of high-attenuation mucus in allergic bronchopulmonary aspergillosis. Chest 2016; 149: 1109-1110.

42 Zemanick ET, Hoffman L, Rosenfeld M. Narrowing in on early cystic fibrosis lung disease. Am J Respir Crit Care Med 2014; 190: 1082-1084.

43 Schwarz C, Bouchara JP, Buzina W, et al. Organization of patient management and fungal epidemiology in cystic fibrosis. Mycopathologia 2018; 183: 7-19.

44 Armstead J, Morris J, Denning DW. Multi-country estimate of different manifestations of aspergillosis in cystic fibrosis. PLOS ONE 2014; 9: e98502.

45 Li DW, Kendrick B. A year-round study on functional relationships of airborne fungi with meteorological factors. Int J Biometeorol 1995; 39: 74-80.

46 Skov M, McKay K, Koch C, et al. Prevalence of allergic bronchopulmonary aspergillosis in cystic fibrosis in an area with a high frequency of atopy. Respir Med 2005; 99: 887-893.

47 Guinea J, Peláez T, Alcalá L, et al. Outdoor environmental levels of Aspergillus spp. conidia over a wide geographical area. Med Mycol 2006; 44: 349-356.

Alshareef F, Robson GD. Prevalence, persistence, and phenotypic variation of Aspergillus fumigatus in the outdoor environment in Manchester, UK, over a 2-year period. Med Mycol 2014; 52: 367-375. Larsen LS. A three-year-survey of microfungi in the air of Copenhagen 1977-79. Allergy 1981; 36: 15-22. Brown K, Rosenthal M, Bush A. Fatal invasive aspergillosis in an adolescent with cystic fibrosis. Pediatr Pulmonol 1999; 27: 130-133.

51 Garczewska B, Jarzynka S, Kus J, et al. Fungal infection of cystic fibrosis patients-single center experience. Pneumonol Alergol Pol 2016; 84: 151-159.

52 Bakare N, Rickerts V, Bargon J, et al. Prevalence of Aspergillus fumigatus and other fungal species in the sputum of adult patients with cystic fibrosis. Mycoses 2003; 46: 19-23.

Harun SN, Holford NHG, Grimwood K, et al. Pseudomonas aeruginosa eradication therapy and risk of acquiring Aspergillus in young children with cystic fibrosis. Thorax 2019; 74: 740-748.

54 Hong G, Psoter KJ, Jennings MT, et al. Risk factors for persistent Aspergillus respiratory isolation in cystic fibrosis. J Cyst Fibros 2018; 17: 624-630.

55 Gago S, Denning DW, Bowyer P. Pathophysiological aspects of Aspergillus colonization in disease. Med Mycol 2019; 57: Suppl. 2, S219-S227.

56 Hatziagorou E, Orenti A, Drevinek P, et al. Changing epidemiology of the respiratory bacteriology of patients with cystic fibrosis-data from the European Cystic Fibrosis Society patient registry. J Cyst Fibros 2020; 19: 376-383.

57 Smith K, Rajendran R, Kerr S, et al. Aspergillus fumigatus enhances elastase production in Pseudomonas aeruginosa co-cultures. Med Mycol 2015; 53: 645-655.

58 Harun SN, Wainwright CE, Grimwood K, et al. Aspergillus and progression of lung disease in children with cystic fibrosis. Thorax 2019; 74: 125-131.

59 Reece E, Segurado R, Jackson A, et al. Co-colonisation with Aspergillus fumigatus and Pseudomonas aeruginosa is associated with poorer health in cystic fibrosis patients: an Irish registry analysis. BMC Pulm Med 2017; 17: 70.

60 Margalit A, Carolan JC, Kavanagh K. Bacterial interactions with Aspergillus fumigatus in the immunocompromised lung. Microorganisms 2021; 9: 435.

61 Patel D, Dacanay KC, Pashley CH, et al. Comparative analysis of clinical parameters and sputum biomarkers in establishing the relevance of filamentous fungi in cystic fibrosis. Front Cell Infect Microbiol 2020; 10: 605241. e01217-e01220.

63 Jhun BW, Jung WJ, Hwang NY, et al. Risk factors for the development of chronic pulmonary aspergillosis in patients with nontuberculous mycobacterial lung disease. PLOS ONE 2017; 12: e0188716.

64 Geurts K, Zweijpfenning SMH, Pennings LJ, et al. Nontuberculous mycobacterial pulmonary disease and Aspergillus co-infection: Bonnie and Clyde? Eur Respir J 2019; 54: 1900117.

65 Bercusson A, Jarvis G, Shah A. CF fungal disease in the age of CFTR modulators. Mycopathologia 2021; 186: 655-664.

66 Currie AJ, Main ET, Wilson HM, et al. CFTR modulators dampen aspergillus-induced reactive oxygen species production by cystic fibrosis phagocytes. Front Cell Infect Microbiol 2020; 10: 372.

Brunel SF, Willment JA, Brown GD, et al. Aspergillus-induced superoxide production by cystic fibrosis phagocytes is associated with disease severity. ERJ Open Research 2018; 4: 00068-2017.

68 lannitti RG, Napolioni V, Oikonomou V, et al. IL-1 receptor antagonist ameliorates inflammasome-dependent inflammation in murine and human cystic fibrosis. Nat Commun 2016; 7: 10791.

69 Culibrk L, Croft CA, Tebbutt SJ. Systems biology approaches for host-fungal interactions: an expanding multi-omics frontier. Omics 2016; 20: 127-138. 
Daly P, Kavanagh K. Pulmonary aspergillosis: clinical presentation, diagnosis and therapy. Br J Biomed Sci 2001; 58: 197-205.

Chabi ML, Goracci A, Roche N, et al. Pulmonary aspergillosis. Daign Intervent Imaging 2015; 96: 435-442.

Desai SR, Hedayati V, Patel $\mathrm{K}$, et al. Chronic aspergillosis of the lungs: unravelling the terminology and radiology. Eur Radiol 2015; 25: 3100-3107.

Schweer KE, Bangard C, Hekmat K, et al. Chronic pulmonary aspergillosis. Mycoses 2014; 57: 257-270.

Maguire CP, Hayes JP, Hayes M, et al. Three cases of pulmonary aspergilloma in adult patients with cystic fibrosis. Thorax 1995; 50: 805-806.

Chow L, Brown NE, Kunimoto D. An unusual case of pulmonary invasive aspergillosis and aspergilloma cured with voriconazole in a patient with cystic fibrosis. Clin Infect Dis 2002; 35: e106-e110.

Felton IC, Simmonds NJ. Aspergillus and cystic fibrosis: old disease - new classifications. Curr Opin Pulm Med 2014; 20: 632-638.

Kosmidis C, Denning DW. The clinical spectrum of pulmonary aspergillosis. Thorax 2015; 70: 270-277.

Buckingham SJ, Hansell DM. Aspergillus in the lung: diverse and coincident forms. Eur Radiol 2003; 13: 1786-1800.

Shoseyov D, Brownlee KG, Conway SP, et al. Aspergillus bronchitis in cystic fibrosis. Chest 2006; 130: 222-226.

Chrdle A, Mustakim S, Bright-Thomas RJ, et al. Aspergillus bronchitis without significant immunocompromise. Ann N Y Acad Sci 2012; 1272: 73-85.

Brandt C, Roehmel J, Rickerts V, et al. Aspergillus bronchitis in patients with cystic fibrosis. Mycopathologia 2018; 183: 61-69.

Hanley-Lopez J, Clement LT. Allergic bronchopulmonary aspergillosis in cystic fibrosis. Curr Opin Pulm Med 2000; 6: 540-544.

Eaton TE, Miller PW, Garrett JE, et al. Cystic fibrosis transmembrane conductance regulator gene mutations: do they play a role in the aetiology of allergic bronchopulmonary aspergillosis? Clin Exp Allergy 2002; 32: 756-761.

Lazarus AA, Thilagar B, McKay SA. Allergic bronchopulmonary aspergillosis. Dis Mon 2008; 54: 547-564.

Knutsen AP, Slavin RG. Allergic bronchopulmonary aspergillosis in asthma and cystic fibrosis. Clin Dev Immunol 2011; 2011: 843763.

Maturu VN, Agarwal R. Prevalence of Aspergillus sensitization and allergic bronchopulmonary aspergillosis in cystic fibrosis: systematic review and meta-analysis. Clin Exp Allergy 2015; 45: 1765-1778.

Carsin A, Romain T, Ranque S, et al. Aspergillus fumigatus in cystic fibrosis: an update on immune interactions and molecular diagnostics in allergic bronchopulmonary aspergillosis. Allergy 2017; 72: 1632-1642.

De Baets F DKL, Van Daele S, Schelstraete P, et al. Risk factors and impact of allergic bronchopulmonary aspergillosis in Pseudomonas aeruginosa-negative CF patients. Pediatr Allergy Immunol 2018; 29: 726-731.

Stevens DA, Moss RB, Kurup VP, et al. Allergic bronchopulmonary aspergillosis in cystic fibrosis - state of the art: Cystic Fibrosis Foundation consensus conference. Clin Infect Dis 2003; 37: Suppl. 3, S225-SS64.

Hiller EJ. Aspergillosis. J R Soc Med 1992; 85: Suppl. 19, 33-35.

Tillie-Leblond I, Tonnel AB. Allergic bronchopulmonary aspergillosis. Allergy Eur J Allergy Clin Immunol 2005; 60: 1004-1013.

Janahi IA, Rehman A, Al-Naimi AR. Allergic bronchopulmonary aspergillosis in patients with cystic fibrosis. Ann Thorac Med 2017; 12: 74-82.

Shah A, Panjabi C. Allergic bronchopulmonary aspergillosis: a perplexing clinical entity. Allergy Asthma Immunol Res 2016; 8: 282-297.

Milla CE, Wielinski CL, Regelmann WE. Clinical significance of the recovery of Aspergillus species from the respiratory secretions of cystic fibrosis patients. Pediatr Pulmonol 1996; 21: 6-10.

Boyle M, Moore JE, Whitehouse JL, et al. The diagnosis and management of respiratory tract fungal infection in cystic fibrosis: A UK survey of current practice. Med Mycol. 2019; 57: 155-160.

Greenberger PA, Bush RK, Demain JG, et al. Allergic bronchopulmonary aspergillosis. J Allergy Clin Immunol Pract 2014; 2: 703-708.

Riscili BP, Wood KL. Noninvasive pulmonary Aspergillus infections. Clin Chest Med 2009; 30: 315-335. 499-507.

9 Rodrigues J, Caruthers C, Azmeh R, et al. The spectrum of allergic fungal diseases of the upper and lower airways. Expert Rev Clin Immunol 2016; 12: 531-550.

0 Eschenhagen P, Grehn C, Schwarz C. Prospective evaluation of Aspergillus fumigatus-specific IgG in patients with cystic fibrosis. Front Cell Infect Microbiol 2020; 10: 602836.

Sunman B, Ademhan Tural D, Ozsezen B, et al. Current approach in the diagnosis and management of allergic bronchopulmonary aspergillosis in children with cystic fibrosis. Front Pediatr 2020; 8: 582964. 
102 Gernez Y, Waters J, Mirković B, et al. Blood basophil activation is a reliable biomarker of allergic bronchopulmonary aspergillosis in cystic fibrosis. Eur Respir J 2016; 47: 177-185.

103 Katelari A, Tzanoudaki M, Noni M, et al. The role of basophil activation test in allergic bronchopulmonary aspergillosis and Aspergillus fumigatus sensitization in cystic fibrosis patients. J Cyst Fibros 2016; 15: 587-596.

104 Mirković B, Lavelle GM, Azim AA, et al. The basophil surface marker CD203c identifies Aspergillus species sensitization in patients with cystic fibrosis. J Allergy Clin Immunol 2016; 137: 436-443.e9.

105 Franquet T, Müller NL, Giménez A, et al. Spectrum of pulmonary aspergillosis: histologic, clinical, and radiologic findings. Radiographics 2001; 21: 825-837.

106 Greene R. The radiological spectrum of pulmonary aspergillosis. Med Mycol 2005; 43: S147-S154.

107 Tiddens HA, Rosenow T. What did we learn from two decades of chest computed tomography in cystic fibrosis? Pediatr Radiol 2014; 44: 1490-1495.

108 Liszewski MC, Ciet P, Lee EY. MR imaging of lungs and airways in children: past and present. Magn Reson Imaging Clin N Am 2019; 27: 201-225.

109 Sodhi KS, Gupta P, Shrivastav A, et al. Evaluation of $3 \mathrm{~T}$ lung magnetic resonance imaging in children with allergic bronchopulmonary aspergillosis: Pilot study. Eur J Radiol 2019; 111: 88-92.

110 Dournes G, Berger P, Refait J, et al. Allergic bronchopulmonary aspergillosis in cystic fibrosis: MR imaging of airway mucus contrasts as a tool for diagnosis. Radiology 2017; 285: 261-269.

111 Garg MK, Gupta P, Agarwal R, et al. MRI: a new paradigm in imaging evaluation of allergic bronchopulmonary aspergillosis? Chest 2015; 147: e58-e59.

112 Aquino SL, Kee ST, Warnock ML, et al. Pulmonary aspergillosis: Imaging findings with pathologic correlation. Am J Roentgenol 1994; 163: 811-815.

113 Jewkes J, Kay PH, Paneth M, et al. Pulmonary aspergilloma: analysis of prognosis in relation to haemoptysis and survey of treatment. Thorax 1983; 38: 572-578.

114 Sansom HE, Baque-Juston M, Wells AU, et al. Lateral cavity wall thickening as an early radiographic sign of mycetoma formation. Eur Radiol 2000; 10: 387-390.

115 Libshitz HI, Atkinson GW, Israel HL. Pleural thickening as a manifestation of Aspergillus superinfection. Am J Roentgenol Radium Ther Nucl Med 1974; 120: 883-886.

116 Barreto MM, Rafful PP, Rodrigues RS, et al. Correlation between computed tomographic and magnetic resonance imaging findings of parenchymal lung diseases. Eur J Radiol 2013; 82: e492-e501.

117 Logan PM, Primack SL, Miller RR, et al. Invasive aspergillosis of the airways: radiographic, CT, and pathologic findings. Radiology 1994; 193: 383-388.

118 Mosquera RA, Estrada L, Clements RM, et al. Early diagnosis and treatment of invasive pulmonary aspergillosis in a patient with cystic fibrosis. BMJ Case Rep 2013; 2013: bcr2013201360.

119 Agarwal R, Chakrabarti A, Shah A, et al. Allergic bronchopulmonary aspergillosis: review of literature and proposal of new diagnostic and classification criteria. Clin Exp Allergy 2013; 43: 850-873.

120 Panchal N, Bhagat R, Pant C, et al. Allergic bronchopulmonary aspergillosis: the spectrum of computed tomography appearances. Respir Med 1997; 91: 213-219.

121 Kalil ME, Fernandes ALG, Curzel ACDS, et al. Allergic bronchopulmonary aspergillosis presenting a glove-finger shadow in radiographic images. J Bras Pneumol 2006; 32: 472-475.

122 Martinez S, Heyneman LE, McAdams HP, et al. Mucoid impactions: finger-in-glove sign and other CT and radiographic features. Radiographics 2008; 28: 1369-1382.

123 Agarwal R, Sehgal IS, Dhooria S, et al. Developments in the diagnosis and treatment of allergic bronchopulmonary aspergillosis. Expert Rev Respir Med 2016; 10: 1317-1334.

124 Occelli A, Soize S, Ranc C, et al. Bronchocele density in cystic fibrosis as an indicator of allergic broncho-pulmonary aspergillosis: a preliminary study. Eur J Radiol 2017; 93: 195-199.

125 Refait J, Macey J, Bui S, et al. CT evaluation of hyperattenuating mucus to diagnose allergic bronchopulmonary aspergillosis in the special condition of cystic fibrosis. J Cyst Fibrosis 2019; 18: e31-e36.

126 Thia LP, Balfour Lynn IM. Diagnosing allergic bronchopulmonary aspergillosis in children with cystic fibrosis. Paediatr Respir Rev 2009; 10: 37-42.

127 Ledoux MP, Toussaint E, Denis J, et al. New pharmacological opportunities for the treatment of invasive mould diseases. J Antimicrob Chemother 2017; 72: Suppl. 1, i48-i58. 